\title{
Pesquisas agronômicas das plantas medicinais da Mata Atlântica regulamentadas pela ANVISA
}

\author{
MING, L.C. ; FERREIRA, M.I.; GONÇALVES, G.G. \\ UNESP-Faculdade de Ciências Agronômicas, Departamento de Produção Vegetal, Setor Horticultura, Rua José \\ Barbosa de Barros,1780, CEP: 18610-307, Botucatu-Brasil linming@fca.unesp.br; Iza_bio@yahoo.com.br; \\ gg.goncalves@uol.com.br
}

\begin{abstract}
RESUMO: Com a divulgação da lista das espécies medicinais pela Agência Nacional de Vigilância Sanitária (ANVISA), de acordo com a Resolução RDC №10, de 09 de março de 2010, o uso dessas plantas passa a ter a chancela oficial do órgão governamental regulamentando seu uso e, em consequência disso, ter sua demanda bastante aumentada. A obtenção desses materiais adquire então grande importância, uma vez que haverá a necessidade de se produzir essas plantas. Com o objetivo de se avaliar a situação das pesquisas agronômicas com essas espécies, particularmente as de ocorrência na Mata Atlântica, foi feito um levantamento do número de publicações a partir dos nomes científicos, na base de dados eletrônica CAB Abstract, de 1990 a 2011. A pesquisa mostrou que o número de publicações por espécie varia de 2 a 1129 , sendo que as espécies com maior número de artigos são aquelas já cultivadas como alimentícias. Das 66 espécies listadas, 36 são exóticas, 24 são da Mata Atlântica e 6 são nativas de outros biomas. Dentre as espécies da Mata Atlântica, foram excluídas as ruderais, frutíferas e arbóreas, devido à maioria dos trabalhos na área agronômica estarem relacionados ao manejo, controle ou produção de frutos e não ao seu cultivo sobre o ponto de vista medicinal. A única exceção foi a espécie medicinal arbórea Maytenus ilicifolia. Assim, foram selecionadas 16 espécies, as quais tiveram as publicações divididas em quatro áreas: Agronomia; Fitoquímica, Ensaios biológicos e Outros. Nesta pesquisa foi possível identificar que $32 \%$ dos artigos publicados são agronômicos, área que apresenta menos publicações do que a área de atividade biológica, que tem $40 \%$ das publicações, e a área de fitoquimica tem $20 \%$ das publicações. Estes resultados mostram que os pesquisadores estão atentos à importância das pesquisas agronômicas com plantas medicinais, mas que se faz necessário realizar trabalhos de domesticação das espécies selvagens e de fitotecnia com as espécies menos estudadas, para viabilizar o cultivo, a conservação dos recursos genéticos vegetais e do meio ambiente.
\end{abstract}

Palavras-chave: Plantas nativas, pesquisas, cultivo, domesticação de espécies, conservação

ABSTRACT: Agronomic research of Atlantic Forest medicinal plants regulated by ANVISA. With the release of the list of medicinal plants by Agência Nacional de Vigilância Sanitária (ANVISA), according to RDC №10, March, 9th , 2010, the use of these plants is replaced by the official seal from the government regulator, in consequence, have greatly increased their demand. The production of these materials then acquires a great importance, as there will be a need to produce these plants. Aiming to assess the status of agronomic research on these species, particularly the species which occur in Atlantic Forest, a survey was made of publications from the scientific names in the CAB Abstract electronic database, ranging from 1990 to 2011 . The survey showed that the number of publications varies from 2 to 1129, and the species with the largest number of articles are those already cultivated as food. Of the 66 species listed, 36 are exotic, 24 are native of the Atlantic Forest and 6 are to other biomes. Among the Atlantic Forest species, were excluded the ruderal, fruit, and tree, due to most studies in Agronomy are related to the management, control or fruit production, and not to the cultivation with the medicinal viewpoint. The only arboreus specie excluded was Maytenus ilicifolia. Thus, we selected 16 species, which had its publications divided into three areas: Agronomy; Phytochemistry and Biological Assays. This research identified that $32 \%$ of published articles are agronomic, area that has less content than the area of biological

Recebido para publicação: setembro de 2011

Aceito para publicação: março de 2012

Rev. Bras. PI. Med., Botucatu, v.14, n.esp., p.131-137, 2012. 
activity, which has $40 \%$ of publications, and the area of phytochemistry have $20 \%$ of publications. These results show that researchers are aware of the importance of agronomic research with medicinal plants, but it is necessary to perform work of domestication of wild species and crop science with the less-studied species, to improve the culture, the conservation of plant genetic resources and the environment.

Key words: Native plants, research, cultivation, species domestication, conservation

\section{INTRODUÇÃO}

Com a divulgação da lista de espécies medicinais pela Resolução RDC №10, de 9 de março de 2010 que dispõe sobre o uso de drogas vegetais junto à Agência Nacional de Vigilância Sanitária (ANVISA, 2010), o uso dessas plantas passa a ter a chancela oficial do órgão governamental regulador, e em conseqüência disso, ter sua demanda bastante aumentada. A obtenção desses materiais vegetais adquire então uma grande importância, uma vez que haverá a necessidade de se produzir essas plantas. A própria Política Nacional de Plantas Medicinais e Fitoterápicos já indicava essa necessidade, com o estabelecimento de diretrizes voltadas à parte agronômica das plantas medicinais (Brasil, 2006; 2009).

Área relativamente recente no complexo de pesquisas envolvendo as plantas medicinais, a Agronomia abrange as atividades técnicas relacionadas à produção dessas espécies, que podem ser disponibilizadas através de algumas alternativas: 1) para as plantas exóticas, serem obtidas por cultivo ou por importação; 2) para as plantas nativas, serem obtidas por extrativismo, manejo sustentável ou por cultivo.

Sobre o cultivo das plantas exóticas é necessário o estabelecimento de experimentos visando verificar a capacidade de adaptação dessas plantas às diferentes condições edafo-climáticas do Brasil. Algumas plantas medicinais já se encontram adaptadas ao clima brasileiro, outras ainda estão sendo avaliadas sobre sua capacidade de adaptação e outras não encontraram aqui, em "terra brasilis", condições adequadas ou favoráveis ao desenvolvimento, tendo então a necessidade de fazer sua importação.

Já entre as plantas nativas, é possível afirmar que, em grande maioria, a forma de obtenção é ainda a da coleta em ambientes de ocorrência natural, podendo, com a crescente demanda, afetar drasticamente a disponibilidade uma vez que não se levam em conta diversos fatores de regeneração, frequência e intensidade da coleta, dentre outros.

Alguns estudos de manejo sustentável com resultados importantes já estão ocorrendo (Reis, 1995; Pavan-Fruehauf, 2000). Destaca-se que, nesses estudos, o tempo exigido para a avaliação consistente é grande, principalmente quando se trata de espécies arbóreas, o que demanda um tempo adicional para o desenvolvimento das técnicas de manejo dessas plantas.

De fato, a pesquisa acerca das técnicas de cultivo estão em fases iniciais, apesar de algumas faculdades de Agronomia e órgãos de pesquisas agronômicas estar realizando diversos ensaios visando a domesticação. Ressalta-se que a grande variabilidade genética intraespecífica pode provocar diferentes resultados, devendo, então, o pesquisador estar atento e consciente dessa situação, que, se por um lado pode parecer desfavorável, sob o ponto de vista da uniformização dos resultados, por outro lado representa alternativas químicas, com potencial de novos usos, uma vez que podem ter substâncias químicas diferentes e interessantes para outras necessidades terapêuticas.

Tanto no Brasil como no mundo, de forma geral, a área de Plantas Medicinais está inserida dentro da ciência da Horticultura (Janick, 1968; Filgueira, 1982). A pesquisa agronômica de plantas medicinais teve início no ano de 1960, e o ensino dessa área nas faculdades de Agronomia ocorreu na Universidade de Brasília, no começo dos anos 1980, se consolidando na década seguinte. Hoje, cerca de $15 \%$ das mais de 60 faculdades agrícolas espalhadas pelo Brasil possuem esse conteúdo técnico nos programas curriculares. Nos Simpósios de Plantas Medicinais do Brasil, a apresentação dos primeiros trabalhos na área estritamente agronômica ocorreu em São Paulo, em 1988, sendo hoje uma área consolidada em todos os eventos com a participação de especialistas que contribuem com informações sobre o setor agronômico das plantas medicinais. Em nível de eventos técnicos agronômicos, a maior parte dos trabalhos em plantas medicinais é apresentada nos Congressos Brasileiros de Olericultura (anuais), cuja sociedade organizadora, a Associação Brasileira de Olericultura (ABH), edita um periódico (Horticultura Brasileira) onde os artigos científicos são publicados. Em 1991, foi fundado o Grupo de Trabalho de Plantas Medicinais, Aromáticas e Condimentares, dentro da $\mathrm{ABH}$, que organiza as discussões temáticas com os associados e outros interessados nessa área em seus Congressos (Ming, 1994). 
Com a crescente demanda das plantas medicinais, ganhando maior importância social econômica e cultural, outros setores da sociedade se organizaram promovendo iniciativas para fortalecer as diversas atividades da cadeia produtiva. Setores que anteriormente não mantinham contato entre si passaram a dialogar com mais frequência. Iniciativas governamentais passaram a ganhar apoio da iniciativa privada e vice-versa. Ações sociais implementadas por ONGs e outras entidades, passaram também a ter maior inserção dentro de programas de governo. Órgãos de fomento passaram a financiar projetos com a temática das plantas medicinais. Há hoje no Brasil uma viva atividade em torno delas.

Devido à grande diversidade vegetal com potencial terapêutico e à diversidade cultural do povo brasileiro, os hábitos incluem desde pequenas ervas até árvores, passando por arbustos e plantas trepadeiras. As partes vegetais utilizadas também são diferentes, podendo ser raízes, folhas, frutos, sementes, cascas, látex, e outras. As necessidades terapêuticas também mudam conforme a região do Brasil.

Isso faz com que as características de produção/obtenção dos materiais vegetais para uso medicinal sejam muito diversas, as quais devem ser analisadas caso a caso e resultam em uma complexidade de aspectos a serem estudados, o que estimula a curiosidade e inventividade da pesquisa e dos pesquisadores, professores e alunos. Sempre situações novas aparecem, demandando novas pesquisas.

Então, quais espécies estudar? Quais as prioritárias? De qual região do Brasil? Para qual atividade terapêutica? Qual a parte vegetal?

Algumas reuniões já foram realizadas em tempos recentes, por iniciativa da EMBRAPA CENARGEN e do Ministério do Meio Ambiente (Vieira et al., 2002), com o intuito de se levantar as espécies prioritárias para estudos de conservação desses recursos genéticos vegetais, por bioma brasileiro, para garantia do uso e fornecimento desses recursos. As listas produzidas são enormes, demonstrando o grande potencial que o país tem e o enorme desafio que ainda está pela frente. As listas referentes à Mata Atlântica envolveram a participação de diferentes instituições de pesquisa, ONGs e Universidades, situadas nos estados onde esse bioma ocorre.

A resolução da ANVISA (2010) se constitui em referencial para as pesquisas agronômicas, que visam colaborar com o fornecimento adequado, em quantidade e qualidade. Considerando que $80 \%$ das plantas medicinais nativas são pertencentes à Mata Atlântica, isto demonstra a importância desse bioma para a pesquisa de plantas medicinais.

O objetivo do trabalho é avaliar a situação das pesquisas agronômicas das espécies medicinais do bioma Mata Atlântica que constam da resolução da ANVISA (2010), a partir de levantamento na base de dados CAB Abstract no período de 1990 a 2011.

\section{MATERIAL E MÉTODO}

A partir da listagem das 66 plantas medicinais divulgada pela ANVISA, 2010 estas foram separadas em três categorias: a) plantas exóticas; b) plantas ocorrentes na Mata Atlântica; c) plantas ocorrentes em outros biomas. As categorias foram pesquisadas a partir dos nomes científicos, pelo título, na base eletrônica de dados CAB Abstract, para se obter o número total de publicações em cada uma delas de 1990 a 2011. Essa base de dados é a que contém artigos científicos que incluem agricultura, solos, pesticidas, horticultura, florestas, veterinária, microbiologia, entomologia, parasitologia, fertilizantes, genética, meio ambiente, botânica, fitoquímica e farmacologia.

Das espécies ocorrentes na Mata Atlântica foram excluídas as ruderais, frutíferas e arbóreas, com exceção de Maytenus ilicifoilia (amplamente utilizada na fitoterapia), a fim de se realizar uma pesquisa mais detalhada, procurando verificar quais espécies têm pesquisas publicadas na área agronômica. As ruderais foram excluídas devido ao fato de a maioria dos trabalhos estar relacionada ao manejo e controle; as frutíferas, devido ao grande número de publicações relacionadas à produção de frutos, e as arbóreas, pela dificuldade na instalação e acompanhamento de experimentos na área agronômica, devido ao longo ciclo de desenvolvimento, o que indicaria número pequeno número de publicações nas espécies medicinais arbóreas.

As publicações levantadas foram divididas em 4 grandes grupos, visando facilitar a compreensão dos resultados: 1) Botânica/Agronomia; 2) Fitoquímica; 3) Ensaios biológicos; 4) Outros. O primeiro abrange estudos de propagação, ecofisiologia, nutrição de plantas e biologia molecular; a segunda contempla estudos de caracterização química de espécies e populações silvestres, a terceira inclui microbiologia, farmacologia e parasitologia e última, as sub-áreas não contempladas anteriormente.

\section{RESULTADO E DISCUSSÃO}

As espécies medicinais publicadas na resolução divulgada pela ANVISA (2010) foram separadas em: exóticas, plantas da Mata Atlântica e plantas de outros biomas. Os resultados mostram que 36 espécies são exóticas, 24 são da Mata Atlântica e 6 são pertencentes a outros biomas brasileiros, de acordo com a Tabela 1. Esses números refletem a diversidade cultural brasileira, formada principalmente por gerações de imigrantes europeus,

Rev. Bras. Pl. Med., Botucatu, v.14, n.esp., p.131-137, 2012. 
africanos e orientais, miscigenados com populações autóctones durante séculos, intercambiando modos de vida, conhecimentos e culturas. As três espécies que mais foram pesquisadas foram Allium sativum (1129 artigos), Eucalyptus globulus (935) e Zingiber officinale (554) e mostram claramente plantas bastante utilizadas para fins alimentícias/ condimentares em todo o mundo e o uso do óleo para fins medicinais.

O fato de o número de espécies da Mata Atlântica ser maior do que o dos outros biomas pode refletir a existência de maior número de pesquisadores envolvidos nas instituições de pesquisa e/ou universidades de regiões onde essa vegetação ocorre, em especial os das regiões sudeste e sul do Brasil. Com as espécies ocorrentes em outros biomas brasileiros, a situação é inversa. Outro fator a ser considerado é o aspecto cultural, o uso pelas populações dessas regiões, parecendo ser corriqueiro em seus diversos usos populares, assim, a partir de levantamentos etnobotânicos, as plantas são identificadas, as partes vegetais e usos são conhecidos e divulgados para outras categorias de cientistas, que então as selecionam para objetos de pesquisa nas sub-áreas de atuação.

Quanto à quantidade de artigos publicados no CAB Abstracts, as plantas exóticas possuem o maior valor, com 7105 trabalhos, seguidas das espécies de ocorrência na Mata Atlântica, com 491 e por fim as espécies de outros biomas, com 284 (Tabela 1). O maior número de espécies exóticas também representa o maior tempo em que elas já vêm sendo estudadas e esforço de pesquisa dedicado a elas, tanto por pesquisadores estrangeiros quanto por brasileiros.

Das plantas exóticas, a erva-doce (Pimpinela anisum) e a arnica (Arnica montana) não encontraram no Brasil condições adequadas ao desenvolvimento,

TABELA 1. Lista de espécies exóticas, nativas da Mata Atlântica e Nativas de outros biomas e número total de artigos publicados na base de dados CAB Abstracts de 1990 a 2011 para cada espécie.

\begin{tabular}{|c|c|c|c|c|c|}
\hline A) Plantas Exóticas & CAB & B) Nativas da Mata Atlântica & CAB & C) Nativas de Outros Biomas & CAB \\
\hline Achillea millefolium & 154 & Achyrocline satureioides & 46 & Justicia pectoralis & 15 \\
\hline Aesculus hippocastanum & 155 & Ageratum conyzoides & 164 & Lippia sidoides & 31 \\
\hline Allium sativum & 1129 & Anacardium occidentale & 426 & Passiflora incarnata & 62 \\
\hline Arctium lappa & 66 & Baccharis trimera & 49 & Paullinia cupana & 50 \\
\hline Arnica Montana & 78 & Bidens pilosa & 155 & Stryphnodendrm adstrigens & 41 \\
\hline Calendula officinalis & 231 & Caesalpinia férrea & 23 & Uncaria tomentosa & 85 \\
\hline Cinnamomum verum & 34 & Casearia sylvestris & 42 & Total & 284 \\
\hline Citrus aurantium & 144 & Cordia verbenacea & 21 & & \\
\hline Curcuma longa & 574 & Echinodorus macrophyllus & 7 & & \\
\hline Cymbopogon citratus & 179 & Erythrina verna & 1 & & \\
\hline Cynara scolymus & 210 & Eugenia uniflora & 75 & & \\
\hline Equisetum arvense & 84 & Lippia alba & 84 & & \\
\hline Eucalyptus globulus & 935 & Maytenus ilicifolia & 84 & & \\
\hline Glycyrrhiza glabra & 206 & Mikania glomerata & 45 & & \\
\hline Hamamelis virginiana & 22 & Momordica charantia & 596 & & \\
\hline Harpagophytum procumbens & 75 & Passiflora alata & 56 & & \\
\hline Illicium verum & 41 & Passiflora edulis & 290 & & \\
\hline Malva sylvestris & 27 & Polygonum punctatum & 4 & & \\
\hline Matricaria recutita & 68 & Psidium guajava & 803 & & \\
\hline Melissa officinalis & 199 & Schinus terebinthifolia & 2 & & \\
\hline Mentha piperita & 196 & Solanum paniculatum & 12 & & \\
\hline Mentha pulegium & 47 & Taraxacum officinale & 197 & & \\
\hline Peumus boldus & 43 & Vernonia condensata & 7 & & \\
\hline Phyllanthus niruri & 82 & Vernonia polyanthes & 7 & & \\
\hline Pimpinela anisum & 94 & Total & 3196 & & \\
\hline Plantago major & 143 & & & & \\
\hline Plectranthus barbatus & 16 & & & & \\
\hline Polygala senega & 11 & & & & \\
\hline Punica granatum & 456 & & & & \\
\hline Rhamnus purshiana & 2 & & & & \\
\hline Rosmarinus officinalis & 368 & & & & \\
\hline Salix Alba & 64 & & & & \\
\hline Salvia officinalis & 261 & & & & \\
\hline Sambucus nigra & 150 & & & & \\
\hline Senna alexandrina & 7 & & & & \\
\hline Zingiber officinale & 554 & & & & \\
\hline Total & 7105 & & & & \\
\hline
\end{tabular}


TABELA 2. Porcentagem de publicações na área agronômica, fitoquímica, ensaios biológicos e outros, de espécies medicinais nativas da Mata Atlântica no CAB Abstracts, de 1990 a 2011.

\begin{tabular}{lccccc}
\hline $\begin{array}{l}\text { Espécies da Mata } \\
\text { atlântica selecionadas }\end{array}$ & CAB & $\begin{array}{c}\text { Agronomia } \\
\text { (\%) }\end{array}$ & $\begin{array}{c}\text { Fitoquímica } \\
\text { (\%) }\end{array}$ & $\begin{array}{c}\text { Ensaios } \\
\text { biológicos (\%) }\end{array}$ & $\begin{array}{c}\text { outros } \\
\text { (\%) }\end{array}$ \\
\hline Achyrocline satureioides & 46 & 21,0 & 32.60 & 36.9 & 9,5 \\
Baccharis trimera & 49 & 48,9 & 6.12 & 38.7 & 6,28 \\
Caesalpinia férrea & 23 & 34,7 & 0,86 & 52,1 & 12,34 \\
Casearia sylvestris & 42 & 9,0 & 19,0 & 66,6 & 5,4 \\
Cordia verbenácea & 21 & 23,0 & 14,2 & 57,1 & 5,7 \\
Echinodorus macrophyllus & 7 & 0,0 & 28,5 & 57,1 & 14,4 \\
Erythrina verna & 2 & 50,0 & 0,0 & 50,0 & 0,0 \\
Lippia Alba & 84 & 34,0 & 40,0 & 16,6 & 9,4 \\
Maytenus ilicifolia & 84 & 20,0 & 25,9 & 38,0 & 15,1 \\
Mikania glomerata & 45 & 33,3 & 6,6 & 55,5 & 5,6 \\
Passiflora alata & 56 & 48,0 & 8,9 & 23,2 & 19,9 \\
Polygonum punctatum & 4 & 25,0 & 0,0 & 75,9 & 0,0 \\
Schinus terebinthifolia & 2 & 50,0 & 50,0 & 0,0 & 0,0 \\
Solanum paniculatum & 12 & 16,6 & 0,0 & 83,6 & 0,0 \\
Vernonia condensata & 7 & 28,0 & 0,0 & 57,1 & 14,9 \\
Vernonia polyanthes & 7 & 28,0 & 0,0 & 42,5 & 30,0 \\
Total & 491 & & & & \\
\hline
\end{tabular}

tendo então a necessidade de importação dessas drogas vegetais, elevando assim o custo destes produtos. Já a camomila (Matricaria recutita) é produzida principalmente no sul e sudeste do país, locais nos quais a espécie encontrou condições ideais de cultivo, tendo se aclimatado há mais de cem anos. (Corrêa Júnior, 1994). Outras espécies, como Achillea millefolium, Arctium lappa, Calendula officinalis, Equisetum arvense, Malva sylvestris, Plantago major e Sambucus nigra, são cultivadas em áreas domésticas ou em pequena escala em regiões brasileiras com clima mais ameno. Espécies aromáticas e condimentares são cultivadas em algumas regiões brasileiras, em nível comercial, como Cymbopogon citratus, Melissa officinalis, Metha piperita, Rosmarinus officinalis e Salvia officinalis, algumas delas com importância econômica para agricultores familiares dessas regiões.

Devido às diferentes condições edafoclimáticas brasileiras as espécies exóticas menos estudadas como a castanha-da-Índia (Aesculus hippocastanum), canela-do-Ceilão (Cinnamomum verum), alcaçuz (Glycyrrhiza glabra), hamamélis (Hamamelis virginiana), garra do diabo (Harpagophytum procumbens), anis estrelado (Illicium verum), malva (Malva sylvestris), boldo-do-Chile (Peumus boldus), polígala (Polygala senega), cáscara sagrada (Rhamnus purshiana), salgueiro (Salix alba) e sene (Senna alexandrina) necessitam de pesquisas que visem promover a aclimatação e/ou o melhoramento genético, a fim de avaliar o potencial produtivo (quantidade e qualidade) dessas espécies, o que demanda longo período de tempo. Ressalta-se ainda que outras pesquisas sobre o cultivo serão necessárias para se estabelecer boas práticas agrícolas para estas plantas.

Dentre as espécies nativas que ocorrem na Mata Alântica, encontram-se espécies exóticas ruderais como picão (Bidens pilosa), melão-de-sãocaetano (Momordica charantia), dente-de-leão (Taraxacum officinale) e mentrasto (Ageratum conyzoides) que são de ocorrência frequüente e muito comuns em diferentes estados brasileiros. Isso representa uma vantagem no que se refere à obtenção dessas plantas, por estarem já aclimatadas, além de serem amplamente conhecidas pelo uso tradicional. Muitas espécies ruderais são consideradas plantas invasoras, plantas daninhas, devido à grande facilidade de se propagarem em ambientes antrópicos ou agricultáveis, havendo inúmeros trabalhos visando estudar a biologia e também o controle, fazendo com que esse número se eleve.

$\mathrm{Na}$ pesquisa realizada na base de dados CAB Abstract o número total de artigos para cada espécie variou de 2 a 1129 (Tabela 1). As espécies que apresentaram alto número de publicações são, além de medicinais, também usadas na alimentação como condimentos e assim têm ampla tradição de cultivo. Muitas são espécies ruderais, tais como, alho (Allium sativum), goiaba (Psidium guajava), cúrcuma (Curcuma longa), gengibre (Zingiber officinalis), caju (Anacardium occidentale), romã (Punica granatum), alecrim (Rosmarinus officinalis), laranja (Citrus aurantium), dente-de-leão (Taraxacum officinale) alcachofra (Cynara scolymus), melão-de-são-caetano (Momordica charantia) e maracujá (Passiflora edulis).

Analisando o número de artigos por área de pesquisa nas 16 espécies da Mata Atlântica 
selecionadas da listagem, a área biológica foi a que apresentou o maior número, 197, representando $40 \%$ do total. Aárea de Agronomia vem a seguir com 158 artigos (32\%), seguida da área de Fitoquímica, com 101 artigos (20\%).

Das espécies encontradas na Mata Atlântica, as cinco espécies que apresentaram maior número de artigos publicados são Lippia alba (84 artigos), Maytenus ilicifolia (84), Passiflora alata (56), Baccharis trimera (49) e Achyrocline satureoides (46). Como já foi dito anteriormente, esses números refletem o uso frequente dessas espécies por populações da região e a priorização nas pesquisas por universidades/órgãos de pesquisas e pesquisadores. Lippia alba apresentou 34 artigos relacionados a fitoquímica (40\%), 29\% na área agronômica e 16,6\% na área de ensaios biológico. Da área agronômica 13 artigos são relacionados a cultivo/propagação, 8 ligados à fisiologia, 4 relacionados ao controle de pragas e doenças através do seu óleo essencial e 1 relacionado à pós-colheita. L. alba é espécie importante sob o ponto de vista medicinal em várias regiões brasileiras e tem sido bastante estudada nos últimos anos. O levantamento mostra ainda que esta espécie tem potencial para uso na agricultura como inseticida e/ou para controle de microrganismos que atacam plantas.

$\mathrm{Na}$ espécie Maytenus ilicifolia, a área com maior número de artigos é a de ensaios biológicos, com 32 artigos (38\%), seguido pela área de fitoquímica (25) e pela área de agronomia (17). Desta última área, destaca-se a sub-área de cultivo/propagação, com 14 artigos. A espinheira-santa tem sido sistematicamente estudada a partir dos finais de 1980 devido ao fato de o Ministério da Saúde ter patrocinado diferentes estudos farmacológicos/ clínicos com plantas medicinais e esta espécie ter apresentado resultados positivos, passando então a ser foco de estudos de diferentes áreas cientíticas. Já na espécie Passiflora alata, a área com maior número de artigos foi a de Agronomia, com 27 (48\%), seguido pela área de atividades biológicas (13\%) e de fitoquímica $(11,1 \%)$. Na área agronômica, a principal sub-área foi a de cultivo/ propagação, com 21 artigos. Isso se justifica pelo fato de que, além de medicinal, a $P$. alata tem uso alimentício.

Diferentemente destas, as espécies que apresentam menor número de trabalhos são conhecidas e utilizadas popularmente apenas como plantas medicinais, algumas delas são arbóreas como o mulungu (Erythrina verna) e o pau ferro (Caesalpinia ferrea) e outras são herbáceas higrófilas como o chapéu de couro (Echinodorus macrophyllus), características estas que podem dificultar o cultivo.

Espécies com ampla distribuição como o assa-peixe (Vernonia polyanthes), e o boldo baiano
(Vernonia condensata) apresentam alta densidade nos locais de ocorrência, o que pode levar ao aumento do extrativismo, podendo-se tornar vantajoso em relação ao cultivo (Reis, 1995). Comparando-se o total de artigos publicados entre os quatro grupos, a área agronômica apresenta valor considerável, mostrando que os pesquisadores estão atentos à importância das pesquisas agronômicas com plantas medicinais. Assim se faz necessário ampliar a realização de trabalhos de domesticação das espécies selvagens para viabilizar o cultivo e a conservação dos recursos genéticos vegetais e do meio ambiente.

Com a regulamentação de uso de certas plantas medicinais como drogas vegetais junto à Agência Nacional de Vigilância Sanitária (ANVISA, 2010) em breve poderá ocorrer um aumento de consumo destas plantas, implicando numa maior demanda de pesquisa agronômica, tanto para espécies exóticas como para espécies nativas que possuem escassas informações agronômicas e botânicas, na busca de se garantir a qualidade e a disponibilidade destas drogas vegetais no mercado.

\section{REFERÊNCIA}

AGÊNCIA NACIONAL DE VIGILÂNCIA SANITÁRIA. Resolução - RDC n.10, de 9 de março de 2010. Dispõe sobre a notificação de drogas vegetais junto à Agência Nacional de Vigilância Sanitária (ANVISA) e dá outras providências. Diário Oficial da União, Brasília, DF, 10 mar. 2010. Seção 1, p.52-59. Disponível em:<http://www.mp.sp.gov.br/portal/page/portal/cao_ consumidor/legislacao/leg_saude/leg_sau_anvs/ Resol-Anvisa.pdf>. Acesso em: 22 abr. 2010.

BRASIL. Ministério da Saúde. Secretaria de Ciência, Tecnologia e Insumos Estratégicos. Departamento de Assistência Farmacêutica e Insumos Estratégicos.

Programa Nacional de Plantas Medicinais e Fitoterápicos / Ministério da Saúde, Secretaria de Ciência, Tecnologia e Insumos Estratégicos, Departamento de Assistência Farmacêutica e Insumos Estratégicos. Brasília, DF.: Ministério da Saúde, 2009. 136p. Disponível em: <http://portal.saude.gov.br/portal/arquivos/pdf/ plantas_medicinais.pdf>. Acesso em: 22 abr. 2010.

BRASIL. Ministério da Saúde. Secretaria de Ciência, Tecnologia e Insumos Estratégicos. Departamento de Assistência Farmacêutica. Política nacional de plantas medicinais e fitoterápicos / Ministério da Saúde, Secretaria de Ciência, Tecnologia e Insumos Estratégicos, Departamento de Assistência Farmacêutica. Brasília, DF: Ministério da Saúde, 2006. 60p. Disponível em: <http:// bvsms.saude.gov.br/bvs/publicacoes/politica_ nacional_fitoterapicos.pdf>. Acesso em: 22 abr. 2010. CORRÊA JÚNIOR, C. Influência das adubações orgânica e química na produção de camomila [Chamomila recutita (L.) Rauschert] e do seu óleo essencial. 1994. 95p. Tese (Mestrado) - Faculdade de Ciencias Agronômicas, UNESP, Botucatu-SP. 
FILGUEIRA, F.A.R. Manual de olericultura: cultura e comercialização de hortaliças. 2.ed. São Paulo: Editora Agronômica Ceres, 1982. 357p.

JANICK, J. A ciência da horticultura. Rio de Janeiro: Livraria Freitas Bastos S.A., 1968. 485p.

MING, L.C. Estudo e pesquisa de plantas medicinais na agronomia. Horticultura Brasileira, v.12, n1, p.3-9, 1994. PAVAN-FRUEHAUF, S. Plantas medicinais de Mata Atlântica: manejo sustentado e amostragem. São Paulo: Annablume/Fapesp, 2000. 216p.

REIS, M.S. Manejo sustentado de plantas medicinais em ecossistemas tropicais. In: Di STASI, L.C. Plantas medicinais: arte e ciência. São Paulo: Editora da Universidade Estadual Paulista, 1996. 230p. VIEIRA, R.F. et al. Estratégias para conservação e manejo de recursos genéticos de plantas medicinais e aromáticas: Resultados da 1a Reunião Técnica. Brasília: Embrapa recursos Genéticos e Biotecnologia/Instituto Brasileiro de Meio Ambiente e dos Recursos Naturais Renováveis (IBAMA)/ Conselho Nacional de Desenvolvimento Científico e Tecnológico (CNPq). 2002, 184p. 